\section{Craniospinal Irradiation in Medulloblastoma using High Energy Medical Linear Accelerator: an Innovative Approach to Planning Technique}

\author{
Gurjar O. P.1,4*, Arya R. ${ }^{2}$ Goyal H. ${ }^{3}$, Handa P. ${ }^{3}$, Bagdare P3, \\ Khan A. ${ }^{3}$, Paliwal R. K. ${ }^{4}$, Mishra S. P.4,5
}

\begin{abstract}
Background: Craniospinal irradiation (CSI) of medulloblastoma poses technological challenges due to the involvement of large treatment volume. Commonly, the whole treatment length is covered with two different isocentric plans in which the junction is shifted after every five fractions to overcome the possibility of hot and cold spot.
\end{abstract}

Objective: This study aims to evaluate dosimetrically and clinically the innovative planning technique for the CSI which doesn't need re-planning and re-setup of patients after every five fractions.

Material and Methods: Computed tomography was done for fifteen (ten children and five adults) patients diagnosed with medulloblastoma. Treatment planning for 36 Gray (Gy) in 20 fractions (\#) at the rate of $1.8 \mathrm{~Gy} / \#$ was done on the treatment planning system. A single plan for children was created with two bilateral fields of 6 Mega Voltage (MV) energy for cranium and one posterior field of $6 \mathrm{MV}$ for spinal cord (C1-S2). Two plans for adult patients were created, first plan was with two bilateral fields of $6 \mathrm{MV}$ for cranium and two posterior oblique fields of $6 \mathrm{MV}$ for cervical and the part of thoracic spinal cord (up to T8-T9). The second plan was with a single posterior field of $15 \mathrm{MV}$ covering remaining thoracic (T8-T9 to T12), lumbar and sacrum (up to lower border of S2) spine. After careful evaluation of all the plans, treatment was delivered; acute toxicities were recorded.

Results: $95 \%$ of prescribed dose was received by more than $95 \%$ of planning target volume in all the plans with the acceptable hot spot and good homogeneity index. All the patients reported common radiation induced acute toxicities (headache, vomiting, weakness) during radiotherapy.

Conclusion: The new planning technique for CSI has acceptable dosimetric and acute clinical possibilities; therefore it can be used for CSI for improved homogeneous dose delivery.

\section{Keywords}

Craniospinal Irradiation, Feathering Technique, Field Edge Matching, Medulloblastoma

\section{Introduction}

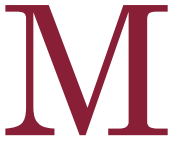

edulloblastoma is the most common childhood malignant brain tumor, it consists of $40 \%$ of all posterior fossa tumors. Median age of presentation is 5-6 years in children and 25 years in adult, the ratio is $2: 1$ for males to females. $30-40 \%$ of patients have craniospinal fluid (CSF) spread at the time of diagnosis [1].
${ }^{1}$ Department of Ra-

diotherapy, All India

Institute of Medical Sci-

ences, Bhopal

${ }^{2}$ Department of Radia-

tion Oncology, Mahatma

Gandhi Memorial Medi-

cal College, Indore

${ }^{3}$ Roentgen-SAIMS Radia-

tion Oncology Centre,

Sri Aurobindo Institute

of Medical Sciences,

Indore

${ }^{4}$ Department of Physics,

Mewar University, Chit-

torgarh

${ }^{5}$ Department of Radia-

tion Oncology, Dr. Ram

Manohar Lohia Institute

of Medical Sciences,

Lucknow, India

*Corresponding author:

O. P. Gurjar

Department of Ra-

diotherapy, All India

Institute of Medical

Sciences,

Bhopal-462020, India

E-mail: ominbarc@gmail. com

Received: 29 April 2017

Accepted: 3 October 2017 
Treatment of medulloblastoma is provided by radiotherapy alone or concurrent chemo -radiotherapy post-surgery. Chemotherapy along with radiation decreases the total curable dose which results in low acute as well late toxicity [2, 3]. Hughes et al. [4] presented in their study that 23.4 Gray (Gy) in 13 fractions (\#) along with chemotherapy gave the results of regional control in 23 of 24 patients when were treated with dose less than 27 Gy (median dose $24 \mathrm{~Gy}$ ), majority of these patients were with T3a-T4 disease.

The standard radiotherapy treatment of high risk medulloblastoma in current era is $36 \mathrm{~Gy}$ in 20\#@1.8Gy/\# to the craniospinal axis followed by 18 Gy boost to posterior fossa [5, 6]. Patients are set to prone position, then two plans with different isocenters are used to treat cranium and whole spinal cord. The first plan covers the cranium and the second one covers the whole spine, the junction of both plans remains at the neck level where the cold spot towards posterior and hot spot towards anterior side are created due to the divergence of the junction fields.

A calculated separation ( $\mathrm{S}$ ) is maintained between cranium field and spinal field so as to optimize the hot and cold spots, this separation is calculated using the formula $\mathrm{S}=0.5 \times$ $\mathrm{L} \times(\mathrm{d} / \mathrm{SSD})$. Where, $\mathrm{L}$ is length of spine field, SSD is "source to surface distance" and $d$ is the "depth" at which both fields to be matched [7]. To manage the hot spot and cold spot, the junction is moved after every $5 \#$ in the longitudinal direction by 0.5 to $1 \mathrm{~cm}$, which is known as feathering technique $[5,7]$.

Michalski et al. [8] presented in their study how to match the cranial and spinal field by rotating couch and collimator to avoid the hot and cold spot and achieve desired dose coverage without any changes in the plan during the course of radiotherapy, however it doesn't explain the planning for adult patients with spine length more than $50 \mathrm{~cm}$.

The junction shift technique is complicated in terms of replanning after every $5 \#$ and the repositioning and verification of the patient on the treatment couch. Moreover, the target coverage is not achieved many times as per desired level of homogeneity at the same time because the skin dose remains high. Underdosing of the target results in the recurrence and poorer prognosis $[9,10]$. While the high dose may cause acute and late toxicities [1113]. The necrosis is one of the most common late toxicities owing to the ,high dose [14]. Therefore, this study has been carried out to develop a planning technique with the optimal target coverage and tolerable doses to organs, and this doesn't require any junctions shifting.

\section{Material and Methods}

Fifteen male patients (ten children and five adults) diagnosed with medulloblastoma (three children and one adult patient with WHO grade III and seven children and four adult patients with grade IV) were evaluated for this study. All the patients were planned for radiotherapy doses of 36 Gy in 20 \# @ $1.8 \mathrm{~Gy} / \#$ with $5 \# /$ week standard protocol and then the dose boosted up to 54 Gy to the posterior fossa $[1,5]$. Two thermoplastic sheets (Orfit), one sheet of 2-clamp for brain and another one of 4-clamp for spine were molded for all the patients in the mold room with keeping the patient in prone position and arms down on the flat base plate (POCL Pvt. Ltd., Mumbai) and prone head rest (POCL Pvt. Ltd., Mumbai). All the patients were simulated on the Siemens SOMATOM Definition AS Scanner (Siemens Medical Systems, Germany) and computed tomography (CT) images of 3 $\mathrm{mm}$ slice thickness were acquired and transferred to the treatment planning system (TPS) Eclipse vs. 8.9 (Varian Medical Systems, Palo Alto, CA) for radiotherapy planning using DICOM protocol 3.0.

Target and normal organs were delineated following the standard practice [1]. Tumor and tumor bed were delineated as gross tumor volume (GTV), cranium and spinal cord (C1 to S2) were delineated as clinical target vol- 
ume (CTV) and a uniform margin of $0.5 \mathrm{~cm}$ was given to CTV for delineating planning target volume (PTV). PTV was divided into two parts; PTV_Brain was for cranium and PTV_Spine for spinal cord (C1 to S2). All the organs at risk (OAR) were also delineated.

\section{Treatment planning for children and adult was done as follows}

For children: Two plans with different isocenters at the spine level were planned. The isocenter of first plan was set on the CT image having cervical vertebra $\mathrm{C} 4-\mathrm{C} 5$, which was mostly three slices $(9 \mathrm{~mm})$ towards head from the CT image having shoulders, while the isocentre for the second one was set in such a way that it's Y1 jaw opens full i.e. $20 \mathrm{~cm}$ and exactly covers the PTV_Spine with $0.5 \mathrm{~cm}$ margin at lumber level so that $\mathrm{Y} 2$ jaw can be opened as minimum as required which helps in less divergence at lower edge of the first plan (i.e. isocentre of plan first).

Single posterior beam of 6 megavoltage (MV) at $0^{\circ}$ gantry angle was used in the second plan, the Y1 jaw was fully opened and covered the PTV_Spine with $0.5 \mathrm{~cm}$ margin at the end of lumber. Y2 jaw was opened to cover the PTV_Spine up to isocentre of the first plan. Multileaf collimators (MLC) in Xdirection confined the PTV_Spine with 0.5 $\mathrm{cm}$ margin. The angle between the line representing Y2 jaw and horizontal line showing the CT level on the sagittal view on TPS was measured using the "angle measuring tool" as shown in Figure 1.

In the first plan, two bilateral (cranial) half beams each of 6 megavoltage (MV) were set to cover the PTV_Brain and PTV_Spine up to isocentre with $\mathrm{Y} 1$ jaw fully closed at isocentre, the collimator of both the fields was rotated with the angle as measured in the second plan. The collimator was rotated in both the bilateral fields to match the field edge at isocentre with the field edge at Y2 jaw of second plan. The angle measurement and field arrangement are shown in Figures 1 and 2.
For adults: Two plans with different isocenters at spine level were planned. The isocenter of first plan was set on the CT image having cervical vertebra $\mathrm{C} 4-\mathrm{C} 5$, which was mostly three slices towards head from the CT image having shoulders. Two bilateral fields were used for whole brain and two posterioroblique fields with $30^{\circ}$ wedge at gantry angles $45^{\circ}$ and $315^{\circ}$ to cover cervical and part of thoracic spine (mostly up to T8-T9). All the four fields were of $6 \mathrm{MV}$ energy. Y2 jaw in the bilateral fields was opened to cover PTV_Brain with $0.5 \mathrm{~cm}$ margin and MLC were confined to PTV_Brain with $0.5 \mathrm{~cm}$ margin in $\mathrm{X}$-direction back covered by X1 and X2 jaws. Y 2 jaw was fully closed while Y1 jaw was opened until 20 $\mathrm{cm}$ in both the oblique fields and MLC were confined to PTV_Spine with $0.5 \mathrm{~cm}$ margin.

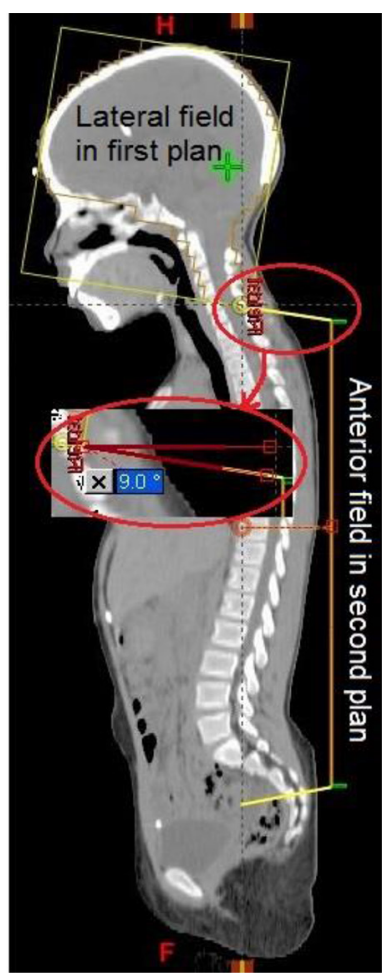

Figure 1: Sagittal view of field arrangement in a plan for one of the children with medulloblastoma. The field matching at $\mathrm{Y} 1$ jaw of the first plan and $\mathrm{Y} 2$ jaw of the second plan was done by rotating the collimators of both the fields in the first plan with the angle measured between $\mathrm{Y} 2$ jaw and the horizontal line. 


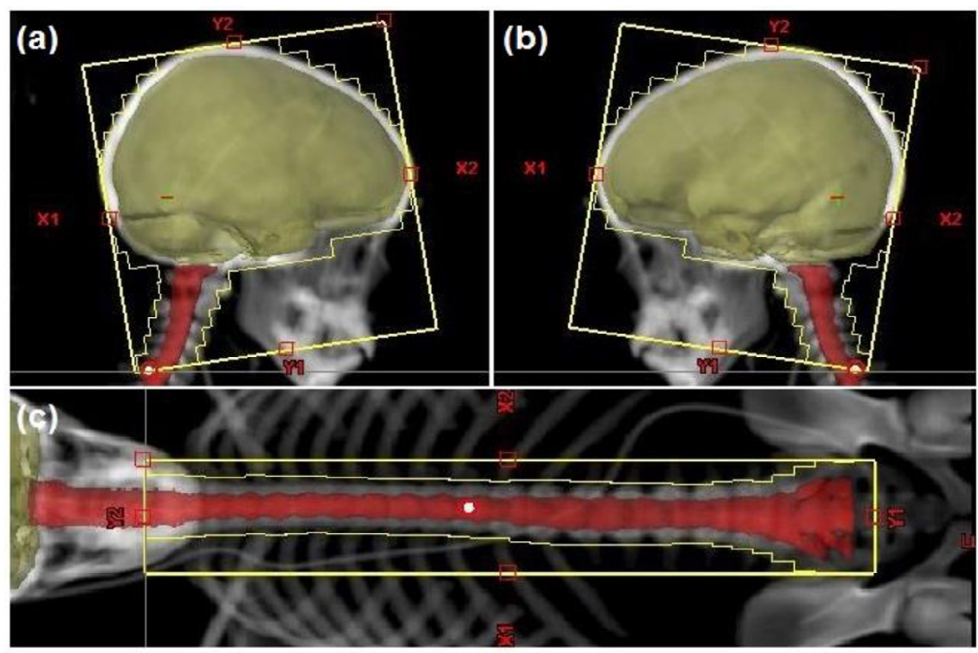

Figure 2: Field eye view of (a) right lateral field and (b) left lateral field in plan first and (c) posterior field in plan second.

The field arrangement is as shown in Figures 3 and 4 .

Isocentre of the second plan was set with same lateral and vertical coordinates as in the first plan and longitudinal coordinate was set in such a way that Y1 jaw of non-coplanar (NCP) field was opened $20 \mathrm{~cm}$ and covered PTV_Spine with $0.5 \mathrm{~cm}$ margin at lumber end. In this plan, the couch and collimators were set to $90^{\circ}$. To decide the gantry angle, the angle between the line representing Y1 jaw of any posterior-oblique fields for the first plan and horizontal line showing the CT level on the sagittal view on TPS was measured using the angle measuring tool. Since in all the patient plans, Y1 jaw of posterior-oblique fields was opened untill $20 \mathrm{~cm}$, therefore this angle in all the plans was same i.e. $8^{\circ}$.

Since the Y2 jaw of NCP field for the second plan is not parallel to the central axis of this field, therefore, gantry will rotate an angle of $8^{\circ}$, Y2 jaw doesn't become parallel to the Y1 jaw of oblique fields of the first plan. As a result, after rotating gantry to $8^{\circ}$, the minor rotation of gantry was performed manually by holding the beam representing line by the mouse in such a way that line representing Y2 jaw became exactly parallel to the $\mathrm{Y} 1$ jaw of oblique field for the first plan first with $2 \mathrm{~mm}$ margin. This margin was given because the radial component for the scatters increased the hot spot at the interface region of both fields. In all the plans of this study, $2 \mathrm{~mm}$ margin was adequate to manage the target coverage with $95 \%$ of PDD and acceptable hot/cold spots.

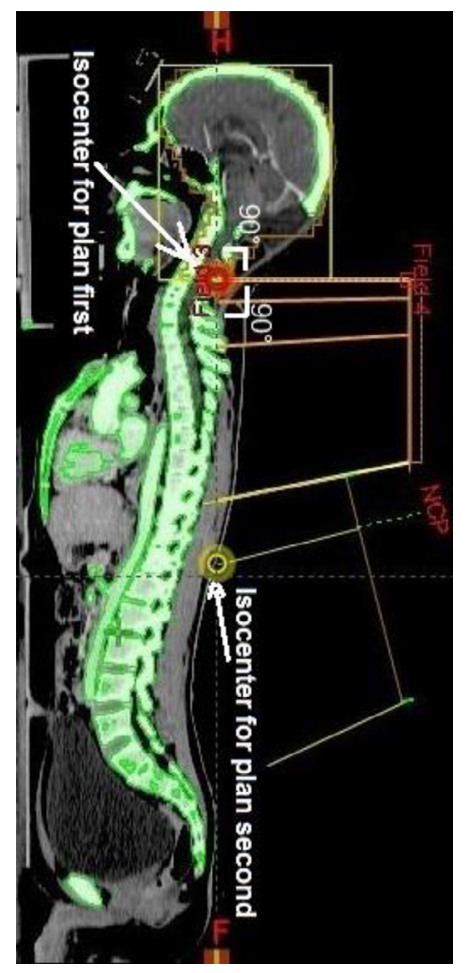

Figure 3: Sagittal view of field arrangement in a plan for one of the adult patients of medulloblastoma. 

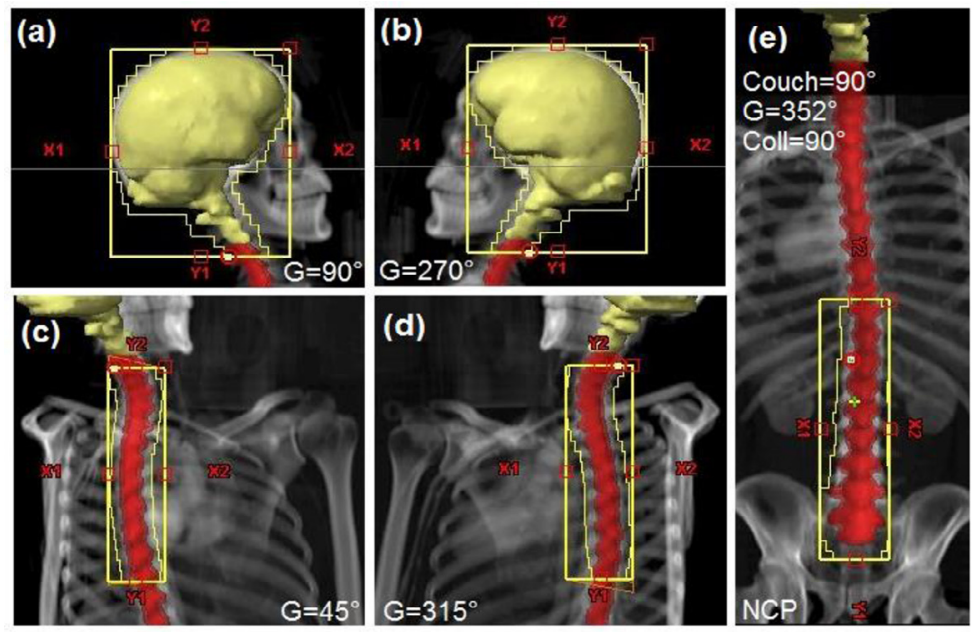

Figure 4: Field eye view of (a) right lateral, (b) left lateral, (c) left posterior oblique (LPO) and (d) right posterior oblique (RPO) fields in the first plan and (e) non coplanar (NCP) field in the second one.

The beam arrangement and beam eye view are as shown in Figures 3 and 4. The angle measurement and Y2 jaw of NCP parallel to Y1 jaw of oblique field are as shown in Figure 5.

In all the plans for children and adult patients, the dose was calculated by using Anisotropic analytical algorithm (AAA) with a grid size of $0.25 \mathrm{~cm}$. Monitoring units (MU) in all fields were adjusted to achieve good target coverage with optimized hot and cold spots. Field-in-field (FIF) was also used in the posterior field of some plans for the children and NCP field of some plans for adult patients wherever required to achieve target coverage with $95 \%$ of the prescribed dose (PD). Target coverage achievement using FIF is as shown in Figure 6.

All the plans were analysed for target coverage, doses to organs at risk (OAR) and homogeneity index (HI). SP34 slab phantom (IBA Dosimetry BmbH, Schwarzenbruck, Germany), 0.13 cc ionization chamber (IBA Dosimetry, Germany) and DOSE1 electrometer (IBA Dosimetry, Germany) were utilized for the point dose verification at the junction of two plans using the standard procedure of patient specific point dose verification using the slab phantom [15]. To get satisfactory dosimetric results, all the plans were approved for treatment of the patients.

All the patients were set on couch of the dual energy linear accelerator (LA) Clinac DMX (Varian Medical Systems, Palo Alto, CA) which is equipped On-Board-Imaging (OBI) system (Varian Medical Systems, Palo Alto, CA). Position verification was done using the standard imaging procedure [16]. All the patients were treated by the plans scheduled for 20\# in fourweeks@5\#/week. Finally, acute toxicities were noted and analysed.

\section{Results}

Plans done for the children and adult patients were based on the same concept and methodology to match the field edges at the junction of two plans. Mean hot spot (maximum dose (Dmax) at any points in the plan) in the PTV_Brain for children, PTV_Brain for adult, PTV_Spine for children and PTV_Spine for adult were found to be $38.63 \mathrm{~Gy}$ (standard deviation (SD): 0.096), 38.76 Gy (SD: 0.24), 38.95 Gy (SD: 0.11) and 39.08 (SD: 0.21) respectively. The mean coverage with $95 \%$ of PD for PTV_Brain for children, PTV_Brain for adult, PTV_Spine for children and PTV Spine for adult were found to be $99.37 \%$ (SD: 


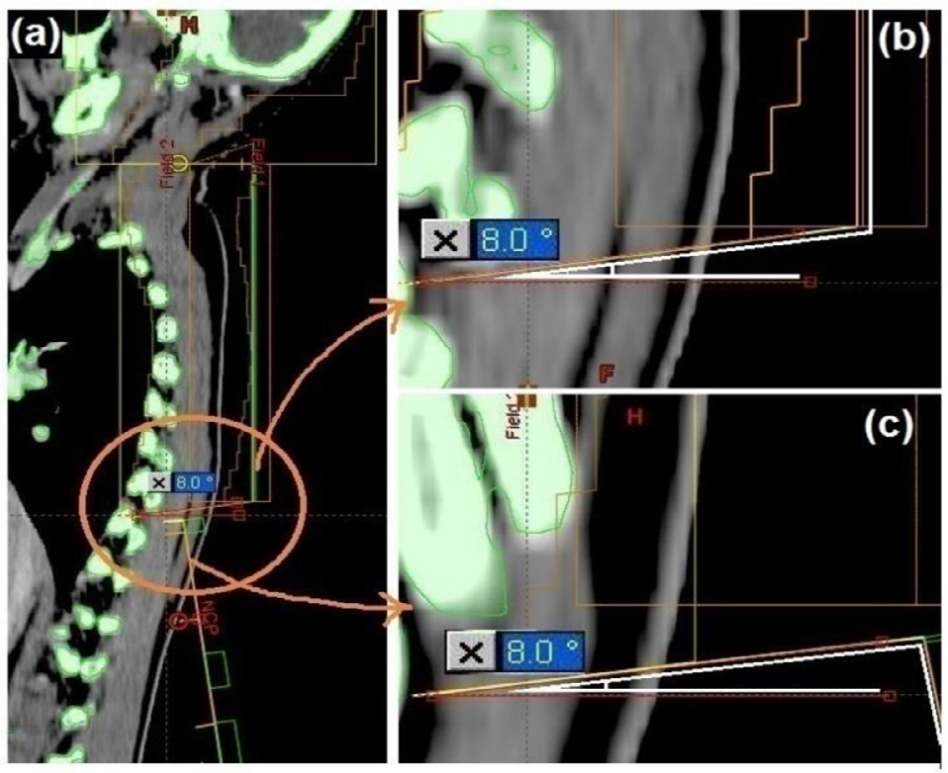

Figure 5: Field edge matching for oblique fields in the first plan and non-coplanar (NCP) field of the second plan are shown for one of the adult patient with medulloblastoma, where (a) the sagittal view of junction fields in the first plan and second plan, (b) the angle measurement between $\mathrm{Y} 1$ jaw for one of the oblique field in first plan and the horizontal line representing the level of CT slice and (c) matching of Y2 jaw of NCP field in the second plan with the Y1 jaw of oblique field in plan the first by rotating the gantry.

0.85), 98.82\% (SD: 0.68), 97.56\% (SD: 0.86) and $96.27 \%$ (SD: 0.98 ). Table 1 describes the mean hot spot and target coverage in the CSI plans for all the fifteen patients.

The dosimetric parameter HI for PTV_Brain and PTV_Spine were found to be 1.07 (SD: 0.03 ) and 1.09 (SD: 0.01) in the plans for children and 1.06 (SD: 0.02) and 1.13 (SD: 0.02) in the plans for adult patients as shown in Table 1 .

The maximum dose (Dmax) and mean dose (Dmean) were noted for all the OAR, and only Dmax was noted for eyes and lenses. The mean Dmax to the right (Rt) eye, left (Lt) eye, Rt lens, Lt lens, trachea, Rt lung, Lt lung, heart, liver, Rt kidney and Lt kidney was found to be 12.76 Gy (SD: 2.72), 11.05 (SD: 1.70), 1.7 Gy (SD: 0.26), 1.41 Gy (SD: 0.25), $30.67 \mathrm{~Gy}$ (SD: 0.66), 33.08 Gy (SD: 0.77), 28.35 Gy (SD: 0.64), 30.27 Gy (0.21), 30.01 Gy (SD: 1.47), 23.05 Gy (SD: 6.98) and 14.42 (SD: $5.57)$ respectively in the plans for children and 10.84 Gy (SD: 3.71), 10.36 (SD: 2.85), 1.66
Table 1: Maximum dose $\left(D_{\text {max }}\right)$, mean dose $\left(D_{\text {mean }}\right)$, planning target volume (PTV) receiving $95 \%$ of prescribed dose $\left(\mathrm{V}_{95}\right)$ and homogeneity index $(\mathrm{HI})$ in the craniospinal irradiation (CSI) plans for ten children and five adult patients.

\begin{tabular}{|c|c|c|c|c|c|}
\hline \multirow{2}{*}{\multicolumn{2}{|c|}{$\begin{array}{l}\text { Dosimetric } \\
\text { parameters }\end{array}$}} & \multicolumn{2}{|c|}{ Children } & \multicolumn{2}{|c|}{ Adults } \\
\hline & & Mean & SD & Mean & SD \\
\hline \multirow{4}{*}{$\begin{array}{l}\text { PTV } \\
\text { Brain }\end{array}$} & $\mathrm{D}_{\max }(\mathrm{Gy})$ & 38.63 & 0.09 & 38.76 & 0.24 \\
\hline & $D_{\text {mean }}(G y)$ & 36.54 & 0.48 & 36.48 & 0.31 \\
\hline & $\mathrm{V}_{95}(\%)$ & 99.37 & 0.85 & 98.82 & 0.68 \\
\hline & $\mathrm{HI}$ & 1.07 & 0.03 & 1.06 & 0.02 \\
\hline \multirow{4}{*}{$\begin{array}{l}\text { PTV }_{-} \\
\text {Spine }\end{array}$} & $D_{\text {max }}(G y)$ & 38.95 & 0.11 & 39.08 & 0.22 \\
\hline & $D_{\text {mean }}(G y)$ & 36.15 & 0.20 & 35.9 & 0.32 \\
\hline & $\mathrm{V}_{95}(\%)$ & 97.56 & 0.86 & 96.27 & 0.98 \\
\hline & $\mathrm{HI}$ & 1.09 & 0.01 & 1.12 & 0.02 \\
\hline
\end{tabular}

Gy: gray; \%: percentage; SD: standard deviation 


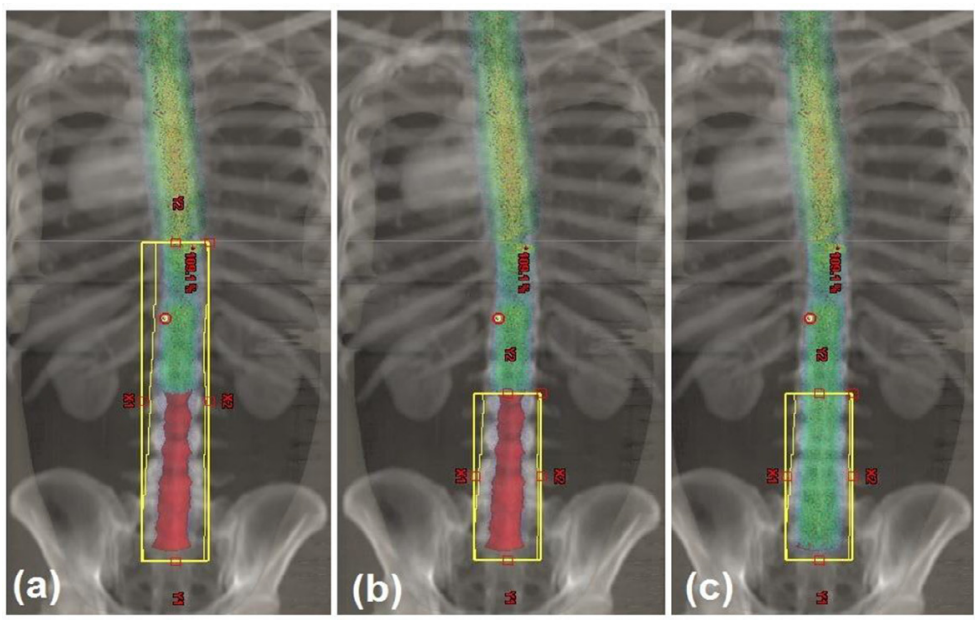

Figure 6: Field eye view of field-in-field (FIF) setup in a plan for one of the adult patient with medulloblastoma, where (a) original non-coplanar (NCP) field of the second plan with $95 \%$ dose color wash, (b) FIF with required MU to cover the volume receiving less than $95 \%$ dose and (c) 95\% dose color wash.

Gy (SD: 0.21), 1.73 Gy (SD: 0.37), 29.06 Gy (SD: 4.35), 31.96 Gy (SD: 3.87), 30.15 Gy (SD: 5.63), 21.38 Gy (SD: 4.86), 29.09 Gy (SD: 3.43), 24.81 Gy (SD: 3.95) and 20.11 (SD: 5.91) respectively, in the plans for adult patients.

The mean Dmean to the trachea, Rt lung, Lt lung, heart, liver, Rt kidney and Lt kidney was found to be 23.09 Gy (SD: 2.52), 4.08 Gy (SD: 1.02), 1.94 Gy (SD: 0.97), 11.63 Gy (SD: 1.35), 4.32 Gy (SD: 0.63), 2.01 Gy (SD: $0.69)$ and 1.28 Gy (SD: 0.35) respectively in the plans for children and 7.95 Gy (SD: 2.39), 7.45 Gy (SD: 2.79), 6.88 Gy (SD: 1.98), 4.07 Gy (SD: 1.49), 5.08 Gy (SD: 1.86), 2.61 Gy (SD: 1.23 ) and 2.22 Gy (SD: 1.65) respectively, in the plans for adult patients. Detailed results have been reported in Table 2 .

Common radiation induced acute toxicities (headache, vomiting, weakness) were reported by all the patients during radiotherapy. Abnormal radiation induced acute toxicities was not reported for any patients treated using this technique.

\section{Discussion}

The primary aim of the planning technique mentioned in this study is to achieve good tar-
Table 2: Doses to organs at risk (OAR) in the craniospinal irradiation (CSI) plans for ten children and five adult patients.

\begin{tabular}{llcccc}
\multicolumn{2}{c}{ OAR } & & \multicolumn{2}{c}{ Children } & \multicolumn{2}{c}{ Adults } \\
\cline { 3 - 6 } & & Mean & SD & Mean & SD \\
\hline Rt eye & $D_{\text {max }}(G y)$ & 12.76 & 2.72 & 10.84 & 3.71 \\
\hline Lt eye & $D_{\text {mean }}(G y)$ & 11.05 & 1.70 & 10.36 & 2.85 \\
\hline Rt eye lens & $D_{\text {max }}(G y)$ & 1.7 & 0.26 & 1.66 & 0.21 \\
\hline Lt eye lens & $D_{\text {mean }}(G y)$ & 1.41 & 0.25 & 1.73 & 0.37 \\
\hline Trachea & $D_{\text {max }}(G y)$ & 30.67 & 0.66 & 29.06 & 4.35 \\
\hline & $D_{\text {man }}(G y)$ & 23.90 & 2.52 & 7.95 & 2.39 \\
\hline Rt lung & $D_{\text {max }}(G y)$ & 33.08 & 0.77 & 31.96 & 3.87 \\
\hline & $D_{\text {mean }}(G y)$ & 4.08 & 1.02 & 7.45 & 2.79 \\
\hline Lt lung & $D_{\text {max }}(G y)$ & 28.35 & 0.64 & 30.15 & 5.63 \\
\hline & $D_{\text {mean }}(G y)$ & 1.94 & 0.97 & 6.88 & 1.98 \\
\hline Heart & $D_{\text {max }}(G y)$ & 30.27 & 0.21 & 21.38 & 4.86 \\
\hline & $D_{\text {mean }}(G y)$ & 11.63 & 1.35 & 4.07 & 1.49 \\
\hline Liver & $D_{\text {max }}(G y)$ & 30.01 & 1.47 & 29.09 & 3.43 \\
\hline & $D_{\text {mean }}(G y)$ & 4.32 & 0.63 & 5.08 & 1.86 \\
\hline Rt kidney & $D_{\text {max }}(G y)$ & 23.05 & 6.98 & 24.81 & 3.95 \\
\hline & $D_{\text {mean }}(G y)$ & 2.01 & 0.69 & 2.61 & 1.23 \\
\hline Lt kidney & $D_{\text {max }}(G y)$ & 14.42 & 5.57 & 20.11 & 5.91 \\
\hline & $D_{\text {mean }}(G y)$ & 1.28 & 0.35 & 2.22 & 1.65
\end{tabular}

Rt: right; Lt: left; Dmax (Gy): maximum dose in gray; Dmean (Gy): mean dose in gray; SD: standard deviation 
get coverage with the minimum dose to OAR at the same time managing the hot spot within acceptable limit so that replanning with junction shift and patient re-setup after all five fractions can be avoided. The planning technique mentioned in the current study has been used in planning for fifteen patients.

The hot spot at the junction of two plans in feathering technique remains very high ( $>$ $115 \%-120 \%$ of the PDD) in the depth, while cold spot within the target remains below $90 \%$ of the PD and this junction region changes in all five fractions $[5,7]$. While in current study, the hot spot in all the plans is about $107 \%$ - $108 \%$ of the PDD and whole target volume is covered with at least $90 \%$ of the PDD. The mean coverage of PTV (brain and spine) in plans for children as well as for adult patients was achieved above $95 \%$ of PDD which is also better than the coverage achieved by the conventional planning techniques. The target coverage and doses to normal organs are comparable with the results presented by Parker et al. [17] and Sharma et al. [18], although Parker et al. did research on short-length patients (spinal length $<38 \mathrm{~cm}$ ) in their study while Sharma et al. did IMRT plans for conventional linear accelerator and helical Tomotherapy for the taller patients.

The doses to both the eyes in all the plans for children as well as for adult patients were well within the tolerance limit (mean dose $<35$ Gy) as mentioned in Radiation Therapy Oncology Group (RTOG) 0225 protocol [19]. Moreover, the doses to both the lenses were minimal as compared to its tolerance value $(<7 \mathrm{~Gy})$ as mentioned in RTOG0539 protocol [20]. The maximum dose to eyes as mentioned in Table 2 is at the very small volume and the scattering contribution is also low for the field edge very near to eye orbits. Similarly the dose to eye lenses is also low because of scattering.

The maximum dose to trachea in the plans for children as well as for adult patients is almost the same and it is high as trachea is a proximal structure to the spine at the neck lev- el, and hence the tracheal wall towards spine received the high dose. The mean dose to the trachea in the plans for adult patients is much lesser as compared to that in plans for the children, it is because two oblique fields were used in case of adult patients for the cervical spine, and the path of these both oblique fields covered the less volume of the trachea which resulted in the less mean dose. While in case of plans for children, exit path of single posterior field passes through trachea. However the doses received by trachea in both kinds of plans were very less.

The maximum dose to both the lungs in the plans for children as well as for adult patients is almost the same, but the mean dose in case of adult patients is comparatively higher as oblique fields pass through lungs and increase the dose to lungs. However, the noted dose in all the plans is very less as compared to the dose constraints for lungs (V20<30\%) as suggested in The Quantitative Analysis of Normal Tissue Effects in the Clinic (QUANTEC) [21]. The dose to heart in the plans of children is higher as compared to adult patients, the reason is again the same as mentioned in above paragraph i.e. the use of oblique fields in case of adult patients, only RAO field is contributing to the dose to heart while LAO is passing through away from heart. In case of the children, the single posterior beam is passing through the part of heart, although the doses in both kinds of the plans are very less as compared to the dose constraints for heart (mean dose $<26$ Gy and V25 < 10\%) [21].

Liver is a very lateral structure and therefore mean dose to it in all types of plans remained very less as compared to its dose constraint (mean dose $\leq 28-32 \mathrm{~Gy}$ ) [21]. The maximum dose to liver is approximately $30 \mathrm{~Gy}$ in all the plans for the less volume. The maximum dose spillage to both the kidneys in all the plans is very little and the mean dose is very less as the kidneys are lateral structures and do not come in the exit path of beam. Owing to the very less margin between kidneys and border 
of the fields, the maximum dose is significant. However, it is found that it can be very less as compared to the tolerance dose for both the kidneys (i.e. mean dose $<15-18$ Gy) [21].

Bladder, rectum and gonads are also critical structures, but beam path was at the sufficient distance from these organs and it was not necessary to report the doses to these structures. However, doses to these structures were seen and analysed on TPS which were found negligible. Only bladder received some significant doses because of its position in divergent path of beam, still it was very less as compared to dose tolerance (V80 $<15 \%$, V75 $<25 \%$, V70 $<35 \%$, V65 <50\%) [21].

The most important feature of this planning technique is to avoid re-planning and resetting the patient after every fraction. When matching the field edges and making the jaws of two neighbor fields parallel need high attention, it is a tedious task to do them in the case of using two oblique fields at cervical and thoracic regions of the plans for adult patients. In the previous sentence, the dose divided into two beams which reduced the exit dose to OARs by $50 \%$ at the same time the target coverage improved while using two fields (at $90^{\circ}$ with each other) with $30^{\circ}$ wedges improved the depth dose in the volume [5, 7]. Although using two oblique fields make difficult match the fields edges at junctions, the required target coverage without unacceptable hot spot and cold spot can be achieved by this special innovative effort.

Since the second plans in case of adult patients were made using one NCP field, after matching the upper jaw of this field with lower jaw of oblique fields of plan 1 , gantry angel became more than $8^{\circ}$, and in this way the source to skin distance (SSD) significantly increased from upper jaw to lower jaw side, because of this differentiated SSD target coverage. It also fell gradually. To achieve the adequate target coverage, FIF was used. Here wedges could resolve the issue of gradually dose reduction, but if the lower jaw was opened more than 10 $\mathrm{cm}$, they would not be allowed to the use of wedges.

\section{Conclusion}

The objective of this study to develop and evaluate an innovative treatment planning which can avoid re-planning and re-setup of the patient, and this has been achieved. Repeated preplanning is a potential source of errors which could be avoided using this technique. Common acute toxicities observed in this study, and dosimetric data indicate that the technique used for planning in current study is worth in terms of clinical benefits as well as planning and setup time.

Based on the clinical observations and dosimetric analysis in current study, it can be concluded that CSI can be done using two bilateral fields with adequate collimator rotation and the posterior fields for the spinal region in the cases where the length of treatment volume covered by posterior field is less than 40 $\mathrm{cm}$, while two bilateral fields for brain, two oblique fields for cervical and thoracic region and one NCP for lower remain under the treatment volume.

\section{Acknowledgment}

We would like to express our thanks to Directors (Dr. S. Hukku and Dr. S. Halder), Roentgen Oncologic Solutions Pvt. Ltd. for permitting and encouraging the study.

\section{Conflict of Interest}

None

\section{References}

1. Hansen EK, Roach M. Handbook of evidence-based radiation oncology. 2nd ed. New York: Springer; 2010.

2. Dennis M, Spiegler BJ, Hetherington CR, Greenberg $M L$. Neuropsychological sequelae of the treatment of children with medulloblastoma. $J$ Neurooncol. 1996;29:91-101. PubMed PMID: 8817420.

3. Halberg FE, Wara WM, Fippin LF, Edwards MS, Levin VA, Davis RL, et al. Low-dose craniospinal radiation therapy for medulloblastoma. Int $J$ Radiat Oncol Biol Phys. 1991;20:651-4. PubMed PMID: 


\section{9.}

4. Hughes EN, Shillito J, Sallan SE, Loeffler JS, Cassady JR, Tarbell NJ. Medulloblastoma at the joint center for radiation therapy between 1968 and 1984. The influence of radiation dose on the patterns of failure and survival. Cancer. 1988;61:19928. PubMed PMID: 3129177.

5. Halperin EC, Brady LW, Perez CA, Wazer DE. Perez \& Brady's principles and practice of radiation oncology. Philadelphia: Lippincott Williams \& Wilkins; 2013.

6. Selek U, Zorlu F, Hurmuz P, Cengiz M, Turker A, Soylemezoglu $F$, et al. Craniospinal radiotherapy in adult medulloblastoma. Strahlenther Onkol. 2007;183:236-40. doi: 10.1007/s00066-0071563-y. PubMed PMID: 17497094.

7. Washington CM, Leaver DT. Principles and Practice of Radiation Therapy: Physics, Simulation, and Treatment Planning. 1st ed. Maryland Heights: Mosby; 2003.

8. Michalski JM, Klein EE, Gerber R. Method to plan, administer, and verify supine craniospinal irradiation. J Appl Clin Med Phys. 2002;3:310-6. doi: 10.1120/1.1508013. PubMed PMID: 12383051; PubMed Central PMCID: PMC5724535.

9. Rieken S, Gaiser T, Mohr A, Welzel T, Witt O, Kulozik AE, et al. Outcome and prognostic factors of desmoplastic medulloblastoma treated within a multidisciplinary treatment concept. BMC Cancer. 2010;10:450. doi: 10.1186/1471-2407-10-450. PubMed PMID: 20731859; PubMed Central PMCID: PMC2939548.

10. Goldwein JW, Radcliffe J, Packer RJ, Sutton LN, Lange B, Rorke LB, et al. Results of a pilot study of low-dose craniospinal radiation therapy plus chemotherapy for children younger than 5 years with primitive neuroectodermal tumors. Cancer. 1993;71:2647-52. PubMed PMID: 8384073.

11. Lawenda BD, Gagne HM, Gierga DP, Niemierko A, Wong WM, Tarbell NJ, et al. Permanent alopecia after cranial irradiation: dose-response relationship. Int J Radiat Oncol Biol Phys. 2004;60:87987. doi: 10.1016/j.ijrobp.2004.04.031. PubMed PMID: 15465206.

12. Jereczek-Fossa BA, Zarowski A, Milani F, Orecchia R. Radiotherapy-induced ear toxicity. Cancer Treat Rev. 2003;29:417-30. PubMed PMID: 12972360.

13. Cairncross JG, Salmon J, Kim JH, Posner JB. Acute parotitis and hyperamylasemia following wholebrain radiation therapy. Ann Neurol. 1980;7:3857. doi: 10.1002/ana.410070419. PubMed PMID:
6155101.

14. Murphy ES, Merchant TE, Wu S, Xiong X, Lukose R, Wright KD, et al. Necrosis after craniospinal irradiation: results from a prospective series of children with central nervous system embryonal tumors. Int J Radiat Oncol Biol Phys. 2012;83:e655-60. doi: 10.1016/j.jijobp.2012.01.061. PubMed PMID: 22768993; PubMed Central PMCID: PMC3529413.

15. Gurjar OP, Mishra SP. A comparative study on patient specific absolute dosimetry using slab phantom, acrylic body phantom and goat head phantom. International Journal of Cancer Therapy and Oncology. 2015;3:3212.

16. Gurjar OP, Mishra SP, Bhandari V, Pathak P, Pant S, Patel P. A study on the necessity of kV-CBCT imaging compared to $\mathrm{kV}$-orthogonal portal imaging based on setup errors: considering other socioeconomical factors. J Cancer Res Ther. 2014;10:5836. doi: 10.4103/0973-1482.139157. PubMed PMID: 25313743.

17. Parker W, Filion E, Roberge D, Freeman CR. Intensity-modulated radiotherapy for craniospinal irradiation: target volume considerations, dose constraints, and competing risks. Int J Radiat Oncol Biol Phys. 2007;69:251-7. doi: 10.1016/j. ijrobp.2007.04.052. PubMed PMID: 17707279.

18. Sharma DS, Gupta T, Jalali R, Master Z, Phurailatpam RD, Sarin R. High-precision radiotherapy for craniospinal irradiation: evaluation of threedimensional conformal radiotherapy, intensitymodulated radiation therapy and helical TomoTherapy. Br J Radiol. 2009;82:1000-9. doi: 10.1259/ bjr/13776022. PubMed PMID: 19581313; PubMed Central PMCID: PMC3473394.

19. RTOG protocol 0225: A phase II study of IMRT +/chemotherapy for nasopharyngeal cancer. Available at: https://www.rtog.org/clinicaltrials/protocoltable/studydetails.aspx?study=0225. Accessed April 09, 2017.

20. RTOG protocol 0539: Phase II Trial of Observation for Low-Risk meningiomas and of Radiotherapy for Intermediate and High-Risk Meningiomas. Available at: https://www.rtog.org/ClinicalTrials/ ProtocolTable/StudyDetails.aspx?ptid=387\& mode $=$ broadcasts $\&$ page $896=2 \&$ study=0539. Accessed April 09, 2017.

21. Marks LB, Yorke ED, Jackson A, Ten Haken RK, Constine LS, Eisbruch A, et al. Use of normal tissue complication probability models in the clinic. Int J Radiat Oncol Biol Phys. 2010;76:S10-9. doi: 10.1016/j.jijrobp.2009.07.1754. PubMed PMID: 20171502; PubMed Central PMCID: PMC4041542. 